\title{
A New Algorithm for Automatic History Matching
}

\author{
W. H. CHEN \\ G. R. GAVALAS \\ J. H. SEINFELD \\ M. L. WASSERMAN
}

\author{
CALIFORNIA INSTITUTE OF TECHNOLOGY \\ PASADENA, CALIF. \\ CHEVRON OIL FIELD RESEARCH CO. \\ LA HABRA, CALIF.
}

\begin{abstract}
History-matching problems, in which reservoir parameters are to be estimated from well pressure data, are formulated as optimal control problems. The necessary conditions for optimality lead naturally to gradient optimization methods for determining the optimal parameter estimates. The key feature of the approach is that reservoir properties are considered as continuous functions of position ratber than as uniform in a certain number of zones. The optimal control approacb is illustrated on a bypothetical reservoir and on an actual Saudi Arabian reservoir, both characterized by single-phase flow. A significant saving in computing time over conventional constant-zone gradient optimization methods is demonstrated.
\end{abstract}

\section{INTRODUCTION}

The process of determining in a mathematical reservoir model unknown parameter values - such as permeability and porosity-that give the closest fit of measured and calculated pressures is commonly called "history matching." In principle, one would like an automatic routine for history matching, applicable to simulators of varying complexity, one that does not require inordinate amounts of computing time to achieve a set of parameter estimates.

In recent years a number of authors have investigated the subject of history matching. ${ }^{1-8}$ All the reported approaches involve dividing the reservoir into a number of zones, in each of which the properties to be estimated are assumed to be uniform. (These zones may, in fact, correspond to the spatial grid employed for the finite-difference solution of the simulator.) Then the history-matching problem becomes that of determining the parameter values in each of, say, $N$ zones, $k_{1}, k_{2}, \ldots, k_{N}$, in such a way that some measure (usually a sum of squares) of the deviation between calculated and

Original manuscript received in Society of Petroleum Engineers office July 3, 1973. Revised manuscript received May 30, 1974 , Paper (SPE 4545) was first presented at the SPE-AIME 48th Annual Fall Meeting, held in Las Vegas, Nev., Sept. 30-Oct. 3, 1973. (C) Copyright 1974 American Institute of Mining, Metallurgical, and Petroleum Engineers, Inc.

1 References listed at end of paper.

This paper will be printed in Transactions volume 257 , which will cover 1974 . observed pressures is minimized. A typical measure of deviation is

$$
\begin{aligned}
J & =\sum_{j=1}^{M} \sum_{i=1}^{n_{j}}\left[p^{\text {obs }}\left(r_{j}, t_{i}\right)\right. \\
& -p^{c a 1} \underset{\sim}{\left.\left(r_{j}, t_{j}\right)\right]^{2}} \ldots, \ldots . . .
\end{aligned}
$$

where $p^{\text {obs }}\left(r_{j}, t_{i}\right)$ and $p^{\text {cal }}\left(r_{j}, t_{i}\right)$ are the observed and calculated pressures at the $j$ th well, which is at location $\underline{r}_{j}=\left(x_{j}, y_{j}\right), j=1,2, \ldots, M$, and where we have $n_{1}$ measurements at Well 1 at $n_{1}$ different times, $n_{2}$ measurements at Well 2 at $n_{2}$ different times, ..., and $n_{M}$ measurements at Well $M$ at $n_{M}$ different times.

To carry out the minimization of Eq. 1 with respect to the vector $k$, most methods rely on some type of gradient optimization procedure that requires computation of the gradient of $J$ with respect to each $k_{i}, i=1,2, \ldots, N$. The calculation of $\partial J / \partial k_{i}$ usually requires, in turn, that one obtain the sensitivity coefficients, $\partial p^{\text {cal }} / \partial k_{i}, i=1,2, \ldots, N$; i.e., the first partial derivative of pressure with respect to each parameter. The sensitivity coefficients can be computed, in principle, in several ways.

1. Make a simulator base run with all $N$ parameters at their initial values. Then, perturbing each parameter a small amount, make an additional simulator run for each parameter in the system. Sensitivity coefficients may then be determined using the finite-difference formula,

$$
\begin{aligned}
& \frac{\partial p^{c a l}(\underset{\sim}{r}, t)}{\partial k_{j}}=\left\{p^{c a l} \underset{\sim}{r}, t ; k_{1}, k_{2}, \ldots,\right. \\
& \left.k_{i-1}, k_{i}+\Delta k_{i}, k_{i+1}, \ldots, k_{N}\right)
\end{aligned}
$$

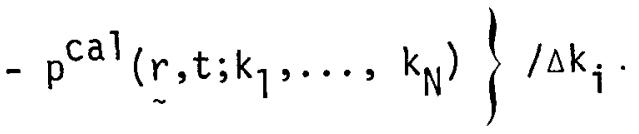

2. Derive a set of "sensitivity equations" from 
the original partial differential equations describing the reservoir system. In this new set of equations in partial differential form, the dependent variables will be the sensitivity coefficients. The finitedifference equivalent of the sensitivity equations will then be solved simultaneously with the original pressure equation. A closely related alternative would be to derive sensitivity equations from the finite-difference form of the simulator equations.

3. Assume that the difference between the observed and calculated pressures is a linear function of the parameters. ${ }^{4}$ That is,

$$
\begin{aligned}
& p^{\text {obs }}\left(r_{j}, t_{j}\right)-p^{c a l}\left(r_{j}, t_{j}\right)=a_{j 0} \\
& +\sum_{i=1}^{N} a_{j i} k_{i} \quad j=1, \ldots, I \text {, }
\end{aligned}
$$

where $l$ is the total number of measurements. Therefore, the sensitivity coefficients become independent of the parameter values. Experience with real problems, however, has indicated that the sensitivity coefficients are not constant over a wide range of parameter values, and consequently, this approach is not a generally valid one for history matching.

4. Compute the sensitivity coefficients by a convolution integral method. ${ }^{1,8}$ This method requires a number of simulations per iteration equal to one plus the number of observation locations.

With $N$ parameters, Method 1 requires $N+1$ simulator runs for each step in the iteration of improving the guesses. Method 2 also requires the solution of $N+1$ partial differential equations per iteration (one simulator run plus $N$ sensitivity equations). Method 4 requires that a number of partial differential equations equal to 1 plus the number of pressure observations be solved per iteration. Herein lies the basic computational inefficiency in the multizonal approach to history matching when $N$ (or $M$ ) is large; namely, the large number of repetitive solutions of partial differential equations that are required in each iteration.

In this paper we propose a new approach to the history-matching problem that is designed to circumvent the excessive computational requirements of standard methods. In essence, we treat the reservoir property being estimated, say permeability, as a continuous function of location rather than as one assuming discrete values in a number of zones. To solve the problem we seek that function - for example, $k(x, y)$ - that minimizes the objective function J. Such an approach is, of course, in keeping with the probable physical nature of an actual reservoir. The key feature of the new approach is that it requires the solution of only two partial differential equations per iteration (one simulator run plus one adjoint equation) regardless of the number of parameters being estimated, the number of wells at which observations are available, and the fineness of the spatial resolution.
The method we present is based on consideration of the history-matching problem as an optimal control problem. The unknown parameter $k(x, y)$, assuming two spatial variables, assumes the role of a control variable that is to be determined so that $J$ is minimized, subject to the constraint that the calculated pressures obey the prescribed reservoir model. From the formulation as an optimal control problem, necessary conditions for optimality in the form of a two-point boundary value problem can be derived. The two-point boundary value problem, the solution of which yields the optimal estimate $k^{*}(x, y)$, cannot be solved analytically in general. Therefore, iterative methods must be used-merhods that often assume the form of gradient optimization methods in which an initial guess $k^{0}(x, y)$ is improved iteratively. The important point is that these iterative methods require the solution of only two partial differential equations per iteration (the original pressure equation and the adjoint equation arising from the necessary conditions for optimality).

We will confine our attention in this paper largely to history matching for single-phase reservoirs. The optimal control approach is equally applicable, in principle, to multiphase flow. In fact, in Appendix $B$ we present the formulation of the optimal control approach for one class of two-phase flow simulators. However, our prime purpose in this paper is to present the derivation of the algorithm and thoroughly test its practical use for single-phase reservoirs.

In the next section the method is summarized. Then we present two computational examples illustrating the use of the method. The first example is a hypothetical reservoir for which the true permeability distribution is assumed known. A detailed comparison of the convergence properties and computational requirements of the new algorithm to two conventional constant zone approaches steepest descent and the Gauss-Newton method is presented for this example. Then, results of application of the new algorithm to an actual reservoir in which both horizontal transmissibilities, $k_{x} b$ and $k_{y} h$, and storage coefficient, $\phi b$, are determined. The full derivation of the algorithm for a single-phase reservoir is presented in Appendix A. In the derivation we assume that the permeability is the unknown parameter. The extension to include estimation of both permeability and porosity is straightforward, and the results are presented in Example 2. As mentioned, Appendix B illustrates the application of the method to one class of two-phase flow simulators.

\section{SUMMARY OF THE ALGORITHM FOR A SINGLE-PHASE RESERVOIR}

Let us consider a reservoir of uniform thickness $b$ but arbitrary cross-section and containing $L$ producing (or injecting) wells and $M-L$ observing wells (with zero production rates). The radius and production flow rate of the $j$ th well will be denoted by $r_{w j}$ and $q_{j}$, respectively. The simulator equation 
for the pressure in a reservoir containing a singlephase fluid can be written (we drop the superscript "cal" for convenience)

$$
\frac{\partial p}{\partial t}=\nabla \cdot(\alpha \underset{\sim}{(r)} \nabla p) \quad \underset{\sim}{r} \in S, \cdots
$$

where $a(r)=k(r) / \phi \mu c$ is the hydraulic diffusivity $(\phi$ is assumed constant), $\nabla$ is the two-dimensional gradient operator with respect to the position vector $r$, and $S$ denotes the region of the plane occupied by the reservoir. $k(r)$ denotes the unknown property, presumed to be the permeability, which is a function of position $r$. Boundary conditions are given on the boundaries $B_{w j}$ of each well and on the external boundary of the reservoir $B_{e}$ as follows:

$$
\frac{\partial p}{\partial l}=0 \quad \quad \sim \varepsilon B_{w_{j}}, j=1,2, \ldots, M
$$

$$
h \int_{B_{w_{j}}} \frac{k(r)}{\mu} \frac{\partial p}{\partial n} d l=
$$$$
\left\{\begin{array}{ll}
q_{j}, & j=1,2, \ldots L \\
0, & j=L+1, \ldots, M
\end{array} .\right.
$$

$$
\frac{\partial p}{\partial n}=0 \quad \underset{\sim}{r} \in B_{e}, \ldots \ldots
$$

where $\partial p / \partial n$ and $\partial p / \partial l$ are the normal and tangential derivatives to the indicated boundary, either $B_{w j}$ or $B_{e}$. Eq. 5 specifies that the pressure on the periphery of each well is uniform, and Eq. 6 specifies the production rate in terms of an integral of the flux. Eq. 7 states that the outer boundary of the reservoir is impermeable. The initial condition to Eq. 4 can be taken as some specified distribution,

$$
p(r, 0)=p_{0}(r)
$$

We have assumed that the reservoir contains $M$ wells at coordinate locations $\underline{r}_{1}, \ldots, \underline{r}_{M}$. The observed pressures at each of the $M$ wells are denoted by $p^{\text {obs }}\left(r_{j}, t_{i}\right), i=1,2, \ldots . ., n_{j}$. The history-matching problem is to determine $k(x)$ to minimize an objective function related to the discrepancy of model and data. A common form of such a function and the one that we shall employ is given by Eq. 1. For the purpose of presenting the algorithm in a general form we shall assume that the objective function is given by

$$
J=\int_{0}^{T} \sum_{j=1}^{M}\left[p^{o b s}\left(r_{j}, t\right)-p\left(r_{j}, t\right)\right]^{2} d t
$$

Eq. 1 can be placed in the form of Eq. 9 as follows:

$$
\begin{aligned}
J= & \int_{0}^{T} \sum_{j=1}^{M} \sum_{i=1}^{n_{j}}\left[p^{\text {obs }}\left(r_{j}, t\right)\right. \\
& \left.-p\left(r_{j}, t\right)\right]^{2} \delta\left(t-t_{i}\right) d t
\end{aligned}
$$

where $\delta($.$) is the Dirac delta function.$

Concisely stated, we wish to minimize $J$, given by Eq. 1, by choice of $k(r)$, subject to Eqs. 4 through 8. This is simply an optimal control problem in a system governed by partial differential equations. The derivation of the necessary conditions for optimality for this problem is presented in Appendix A. If the optimal value of $k(r)$ is denoted $k^{*}(r)$, the necessary conditions for optimality are given by

$$
\begin{array}{ll}
\frac{\partial p}{\partial t}=\nabla \cdot(\alpha *(\underset{\sim}{r)} \nabla p) & \quad r \varepsilon S, \ldots(11) \\
\frac{\partial p}{\partial l}=0 & \underset{\sim}{r} \varepsilon B_{W_{j}} j=1,2, \ldots, M
\end{array}
$$

$$
\begin{aligned}
& h \int_{B_{w_{j}}} \frac{k^{*}(\underset{\sim}{r})}{\mu} \frac{\partial p}{\partial n} d l= \\
& \left\{\begin{array}{l}
q_{j}, j=1,2, \ldots, L \\
0, j=L+1, \ldots, M
\end{array}, \ldots .\right.
\end{aligned}
$$

$$
\begin{aligned}
& \frac{\partial p}{\partial n}=0 \quad \quad r \varepsilon B_{e}, \cdots \cdots \\
& p(r, 0)=p_{0}(r) \quad \underset{\sim}{r} \in S, \ldots \\
& \left.\frac{\partial \psi}{\partial t}=-\nabla \cdot\left(\alpha^{*} \underset{\sim}{r}\right) \nabla \psi\right) \quad \underset{\sim}{\sim} \varepsilon S \\
& \frac{\partial \psi}{\partial l}=0 \quad r_{\varepsilon} \in B_{w_{j}}, \quad j=1,2, \ldots, M
\end{aligned}
$$




$$
\begin{aligned}
& h \int_{B_{W_{j}}} \frac{k^{*}(r)}{\mu} \frac{\partial \psi}{\partial n} d \ell=2 h \phi c\left[p^{o b s}(r, t)\right. \\
& -p(r, t)] \quad \underset{\sim}{\sim} \in B_{W_{j}}, j=1,2 \ldots, M \\
& \frac{\partial \psi}{\partial n}=0 \quad{ }_{\sim} \in B_{\mathrm{e}}, \ldots . . . . \\
& \psi(r, T)=0, \cdot . \cdot . \cdot . \cdot \cdot \cdot \\
& \int_{0}^{T}(\nabla \psi) \cdot(\nabla p) d t=0 .
\end{aligned}
$$

Since the optimal value of $k(r)$ cannot be determined analytically, an iterative solution of the optimal control problem is necessary. Some of the most effective types of iterative methods for the solution of optimal control problems are those in which an initial guess of the control variable, $k^{0}(r)$, is iteratively improved by

$$
k^{j+1}(r)=k^{j}(r)+\delta k^{j}(r) \text {. }
$$

The left-hand side of Eq. 21 is the functional derivative of $J$ with respect to $k(r), \delta J / \delta k(r)$. Only at the optimal solution does Eq. 21 hold. However, $\delta J / \delta k(r)$ given by the left-hand side of Eq. 21 can serve as the means of computing $\delta k(r)$ in an iterative gradient method. Two of such methods are now presented.

\section{METHOD OF STEEPEST DESCENT}

In Appendix $A$ it is shown that

$$
\begin{gathered}
\delta J=-\iint_{S}\left\{\int_{0}^{T}[(\nabla \psi) \cdot(\nabla p)] d t\right\} \\
\cdot \delta \alpha(r) d S .
\end{gathered}
$$

To decrease $J$ by choice of $\delta a(r)$ we want $\delta J<0$. This can be accomplished simply by setting

$$
\delta \alpha(r)=\underset{\sim}{W(r)} \int_{\sim}^{T}[(\nabla \psi) \cdot(\nabla p)] d t, .
$$

or, in terms of $k(r)$,

$$
\delta k \underset{\sim}{r})=\phi \mu \mathrm{CW}(\underset{\sim}{r}) \int_{0}^{T}[(\nabla \psi) \cdot(\nabla p)] d t
$$

where $W(r)$ is an arbitrary positive function of $r$.

From the definition of the function derivative and from Eq. A-19 we can see that we define the perturbations in order to seek the minimum of the objective function by moving the parameter along the gradient of the objective function with respect to the parameter from one iteration to the next. The size of the step-i.e., how far one must proceed along the gradient in each iteration - is controlled by the choice of the weighting factor $W(r)$ in Eq. 24. One method of choosing the weighting factor is to minimize the objective function along the direction of the gradient with respect to the weighting factor, generally necessitating a onedimensional search. The step size can also be determined from the second variation of the objective function. In the former method, we are required to solve the state equation several times. In the latter method, the calculation of the second variation of $J$ is needed. As pointed out previously, a considerable portion of computational time in each iteration in the parameter estimation is devoted to determining the solutions of the state and the adjoint equations. Although determining the weighting factor by the optimization procedures usually reduces the number of total iterations, the computational efforts can be considerable. Thus, in this study, the weighting factor is chosen for the first iteration so that the perturbation will be some percentage of the initial guess value of the parameter (say 30 percent). If the new estimate increases rather than decreases the objective function, the minimum has been overstepped. We then go back to the value from the previous iteration and decrease the weighting factor by a factor of two before forming the new estimate. If the new estimate decreases the objective function, the value of the weighting factor is tripled for the next iteration.

To increase the rate of convergence, a more effective gradient method such as the conjugate gradient method may be employed instead of the method of steepest descent. This method will be discussed in the next section.

The use of Eq. 24 to compute $\delta k(r)$ in conjunction with Eq. 22 constitutes the method of steapest descent. The algorithm is employed as follows:

1. Make an initial guess $k^{0}(r)$ and solve Eqs. 4 through 8 . Evaluate $J$ by Eq. 1 . Select $W(r)$.

2. Compute $\psi(r, t)$ by solving Eqs. 16 through 20 from $t=T$ to $t=0$ using $k^{0}(r)$ and $p^{0}(r, t)$ from Step 1 .

3. Compute $\delta k(r)$ by Eq. 24 and update $k j(r)$ by Eq. 22.

4. If a convergence criterion

$$
\frac{J^{i}-J^{i+1}}{J^{i}} \leq \epsilon
$$

is met, stop; if not, return to Step 1 with $k^{j+1}$ in 
place of $k^{0}$.

\section{CONJUGATE GRADIENT METHOD}

The conjugate gradient method is similar to the method of steepest descent except for the manner in which $\delta k^{j}(r)$ in Eq. 22 is determined. In this method $\delta k j(r)$ is computed by

$$
\delta k^{j}(r)=\beta_{j} S_{j}, \ldots . . . . . .
$$

where

$$
S_{j}=
$$

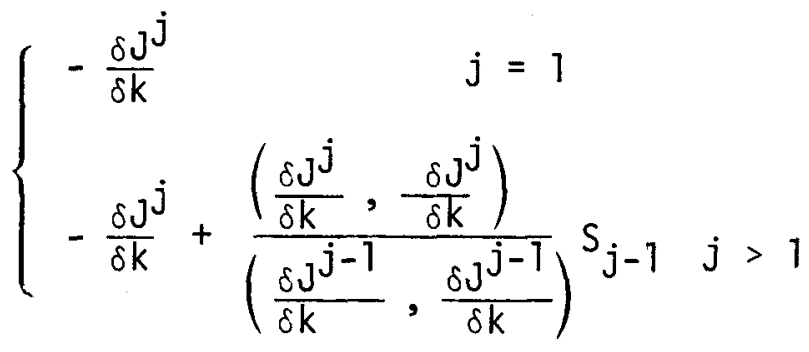

and where the inner product $(a, b)$ is defined as

$$
(a, b)=\int_{0}^{T} \int_{S} a^{T} b d x d y d t .
$$

The scalar $\beta_{j}$ is determined by a one-dimensional search to minimize $J\left(k^{j}+\beta_{j} S_{j}\right)$.

\section{APPLICATION OF THE METHOD}

\section{EXAMPLE 1}

We consider first the estimation of the permeability distribution $k(x, y)$ in the single-phase reservoir shown in Fig. 1, the pressure in which is governed by

$$
\begin{gathered}
\frac{\partial p}{\partial t}=\frac{1}{c \phi \mu}\left[\frac{\partial}{\partial x}\left(k(x, y) \frac{\partial p}{\partial x}\right)\right. \\
\left.+\frac{\partial}{\partial y}\left(k(x, y) \frac{\partial p}{\partial y}\right)\right], \ldots . . \\
p(x, y, 0)=p_{0} \quad 0 \leq x \leq L_{1}, \\
\quad 0 \leq y \leq L_{2} . . . \\
\frac{\partial p}{\partial x}=0 \quad 0, L_{1}, \ldots . \\
\frac{\partial p}{\partial y}=0 \quad . .
\end{gathered}
$$

$h \int_{B} \frac{k}{\mu} \frac{\partial p}{\partial n} d \ell=q \quad$ (injection well)

$$
\begin{gathered}
h \int_{B_{j}} \frac{k}{\mu} \frac{\partial p}{\partial n} d l=0 \quad \text { (observing wells) } \\
j=1,2, \ldots, 8
\end{gathered}
$$

The locations of the injection and observation wells are shown in Fig. 1. The true but presumed unknown permeability was chosen as

$$
k(x, y)=0.02+\frac{0.7}{L_{1}} \sqrt{x^{2}+y^{2}} .
$$

Observed pressures were generated by solving Eqs. 26 through 31 numerically with Eq. 32 and the following parameter values: $\phi=0.2, b=1 \mathrm{ft}, L_{1}$ $=60,000 \mathrm{fr}, L_{2}=30,000 \mathrm{ft}, c=1.72 \times 10^{-5} \mathrm{psi}^{-1}$, $\mu=0.352 \mathrm{cp}, p_{o}=0$, and $q=500 \mathrm{cu} \mathrm{ft} \mathrm{day}{ }^{-1}$ (injection). The alternating-direction implicit method was used to solve the state $(p)$ and adjoint $(\psi)$ equations. ${ }^{11}$

The problem is to estimate $k(x, y)$ from the observed pressures. The objective function used is

$$
\begin{aligned}
J= & \sum_{i=1}^{K} \sum_{j=1}^{M}\left[p^{\text {obs }}\left(x_{j}, y_{j}, t_{j}\right)\right. \\
& \left.-p\left(x_{j}, y_{j}, t_{j}\right)\right]^{2}, \ldots \ldots
\end{aligned}
$$

where $K$ discrete time measurements $(K=20)$ are assumed to be available at each of the eight ( $M$ $=8$ ) observation wells. Note that no pressure data are taken at the injection well.

We shall approach the estimation of $k(x, y)$ in two basic ways - a constant-zone approach and the new algorithm developed in this paper. For the first method it is necessary to divide the reservoir into zones in each of which the permeability is taken to

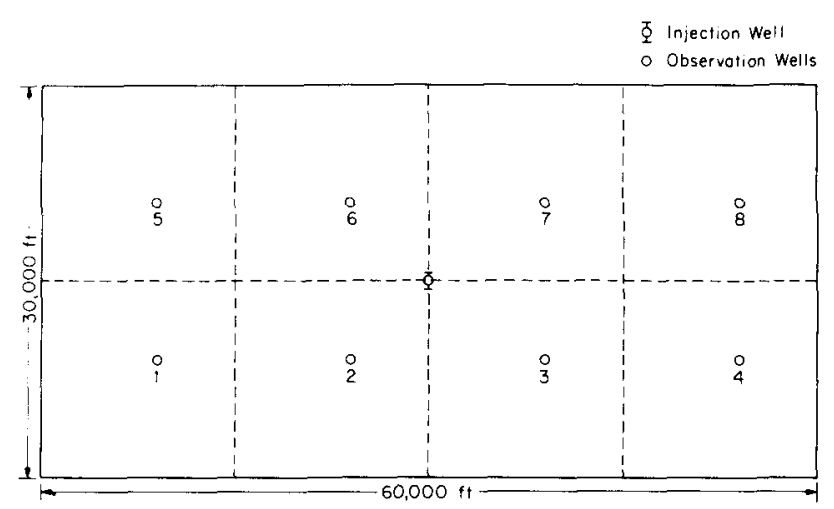

FIG. 1 - HYPOTHETICAL RESERVOIR WITH SINGLE INJECTION WELL AND EIGHT OBSERVATION WELLS DIVIDED INTO EIGHT ZONES. 
be constant. We shall consider two cases, four zones and eight zones, assuming that $k$ is constant in each zone. Thus, we have four unknown parameters in the four-zone case and eight unknown parameters in the eight-zone case. The configuration of the eight zones is shown in Fig. 1, and Fig. 2 shows the location of the four zones. With the constant-zone approach we shall employ two standard parameter estimation methods: (1) the method of steepest descent, and (2) the GaussNewton method. These two methods are now summarized.

In the steepest-descent method, the $(j+1)$ st iterate of $k_{i}, k_{i}^{j+1}$, is determined from

$$
k_{i}^{j+1}=k_{j}^{j}+\gamma \frac{\partial J}{\partial k_{i}}
$$

where $\gamma<0$ and $\partial J / \partial k_{i}$ can be determined from Eq. 9 by

$$
\begin{aligned}
\frac{\partial J}{\partial k_{i}} & =-2 \int_{0}^{T} \sum_{j=1}^{M}\left[p^{o b s}\left(r_{j}, t\right)\right. \\
& -p^{c a l} \underset{\sim}{\left.\left(r_{j}, t\right)\right]} \frac{\partial p^{c a l}\left({\underset{\sim}{j}}_{j}, t\right)}{\partial k_{i}} d t .
\end{aligned}
$$

In the Gauss-Newton method, on the other hand, we assume that each $k_{i}$ differs from the initial guess by an amount $\Delta k_{i}$ and that the calculated pressure can be expanded in a Taylor series about the initial guess,

$$
\begin{aligned}
& p^{c a l}\left(r_{j}, t ; k_{1}+\Delta k_{1}, \ldots, k_{N}+\Delta k_{N}\right) \\
& =p^{c a l} \underset{\sim}{\left.r_{j}, t ; k_{1}, \ldots, k_{N}\right)} \\
& +\sum_{\ell=1}^{N} \frac{\partial p^{c a l}\left({\underset{\sim}{j}}_{j}, t ; k_{1}, \ldots, k_{N}\right)}{\partial k_{\ell}} \Delta k_{\ell} .
\end{aligned}
$$

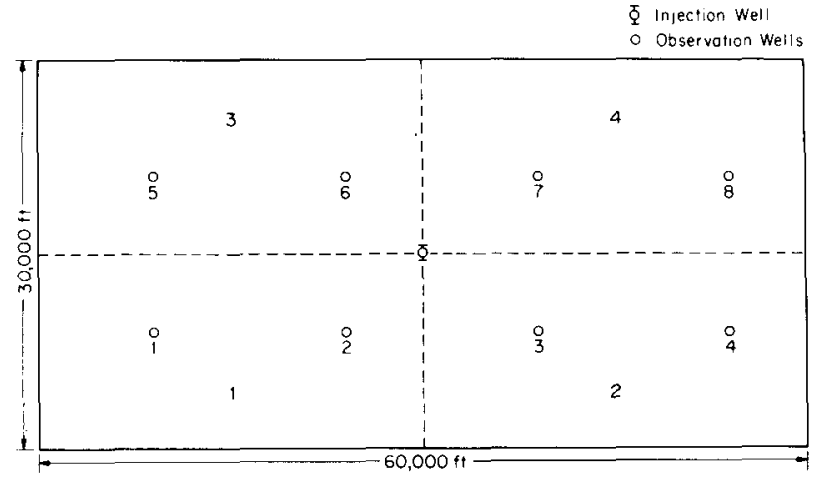

FIG. 2 - HYPOTHETICAL RESERVOIR WITH SINGLE INJECTION WELL AND EIGHT OBSERVATION WELLS DIVIDED INTO FOUR ZONES.
We can substitute Eq. 36 into Eq. 9, making $J$ a function of the $\Delta k_{i}$. Then setting $\partial J / \partial \Delta k_{i}=0$ yields $N$ simultaneous linear equations for the $\Delta k_{i}$, the solution of which yields the recursion formula

$$
k_{i}^{j+1}=k_{i}^{j}+\gamma\left(R^{-1}\right)_{i}\left(\frac{\partial J}{\partial k}\right)^{\top}, .
$$

where $\left(R^{-1}\right)_{i}$ is the $i$ th row of the $N \times N$ matrix $\underset{\sim}{R}$, where the $i, l$ element of $R$ is defined by

$$
\begin{aligned}
R_{i \ell}= & \left(2 \int_{0}^{T} \sum_{j=1}^{M} \frac{\partial p^{c a l}\left({\underset{\sim}{j}}_{j}, t\right)}{\partial k_{i}}\right. \\
& \left.\cdot \frac{\partial p^{c a l}\left({\underset{\sim}{j}}_{j}, t\right)}{\partial k_{\ell}} d t\right), .
\end{aligned}
$$

and $\partial J / \partial k_{N}=\left[\left(\partial J / \partial k_{1}\right),\left(\partial J / \partial k_{2}\right), \ldots,\left(\partial J / \partial k_{N}\right)\right]$, an $N$-dimensional row vector.

We note that $R$ represents an approximation to $H$, where $H$ is the Hessian matrix. This can be seen from the definition of Hessian matrix:

$$
\begin{aligned}
& H_{i \ell}=\frac{\partial^{2} J}{\partial k_{i} \partial k_{\ell}} \\
& =\left(2 \int_{0}^{T} \sum_{j=1}^{M} \frac{\partial p^{c a l}\left(r_{j}, t\right)}{\partial k_{j}} \frac{\partial p^{c a l}\left(\underline{\sim}_{j}, t\right)}{\partial k_{\ell}} d t\right. \\
& -2 \int_{0}^{T} \sum_{j=1}^{M}\left[p^{o b s}\left(r_{j}, t\right)\right. \\
& \left.-p^{c a l} \underset{\sim}{\left.\left(r_{j}, t\right)\right]} \frac{\partial^{2} p^{c a l}\left(\underline{\sim}_{j}, t\right)}{\partial k_{j} \partial k_{\ell}} d t\right) .
\end{aligned}
$$

If the second term is neglected, in the hope that the residue is small, then $R$ is a good approximation to $H$. When $R=H$, we have the so-called Newton method. ${ }^{9}$ The high cost of computing second-order derivatives leads one to make use of simplifications of the Newton method, such as the Gauss-Newton method. Reported computational results indicate that the Gauss-Newton method is one of the most efficient gradient methods. ${ }^{10}$ Sensitivity coefficients for the steepest-descent and Gauss-Newton methods were computed numerically by $\mathrm{Eq}$. 2 by changing each of the $k_{i}$ one at a time by 5 percent of its value and then calculating the corresponding change 
in $p$.

The new algorithm developed here will be applied using both the steepest-descent method of Eqs. 22 and 24 and the conjugate-gradient method of Eqs. 22 and 25 . Therefore, the estimation of $k(x, y)$ was carried out using four different methods, two based on the constant-zone formulation and two based on the new optimal control algorithm.

Some typical results are summarized in Table 1. Of particular interest in the comparison among the four methods are two points:

1. The performance of the method; i.e., how rapidly $J$ was decreased.

2. The computing time requirements of the method-i.e., how many seconds of computing time were required per iteration and for comparable reductions in the objective function $J$.

We see that each of the four methods reduced $J$ by a factor of about 100 in 10 or fewer iterations. However, from the standpoint of computing time, the steepest-descent version of the optimal control algorithm was superior to each of the constant-zone methods in the cases of both four and eight zones. Since the final value of $J$ is different from each method, for the purpose of comparison we can choose a value of $J$, say 13 , at which to compare the methods. Since the number of iterations needed to reach a given value of $J$ is different for different initial guesses, the time reported will be the average time for $k^{0}=0.2$ and $k^{0}=0.7$. Under these conditions the steepest-descent version of the optimal control algorithm takes 55 seconds (five iterations for $k^{0}=0.2$ and three iterations for $k^{0}$ $=0.7$ ); the Gauss-Newton method takes 77 seconds (three iterations for $k^{0}=0.2$ and five iterations for $k^{0}=0.7$ ) for the four-zone case and 100 seconds (two iterations for $k^{0}=0.2$ and five iterations for $\left.k^{0}=0.7\right)$ for the eight-zone case; and the steepest- descent method takes 137 seconds (ten iterations for $k^{0}=0.2$ and five iterations for $\left.k^{0}=0.7\right)$ for the four-zone case and 144 seconds (five iterations for both $k^{0}=0.2$ and $k^{0}=0.7$ ) for the eight-zone case.

Figs. 3 and 4 compare for the Observation Wells 2 and 8 the observed and calculated pressures for the initial guess, the 10th iteration for the steepest-descent version of the optimal control algorithm, and the 10th iteration for the method of steepest descent for the four-zone case. The final pressures for the eight-zone case by the methods of steepest descent and Gauss-Newton are approximately the same as the final pressures calculated by the variational method. The final pressures for the four-zone case by the methods of steepest descent and Gauss-Newton are approximately the same. As we expected, the eight-zone case is superior to the four-zone case in terms of pressure-matching. This can be seen from Figs. 3 and 4.

Figs. 5 and 6 present the distribution of permeability at $y=9,000 \mathrm{ft}$ and $21,000 \mathrm{ft}$ for the initial guess, the 10th iteration for the steepestdescent version of the optimal control algorithm, the 10th iteration for the eight-zone and four-zone cases by the method of steepest descent, and the average value of the true permeability in each zone. The average value in each zone is defined by

$$
k_{i}=\frac{A_{i} \int k(x, y) d x d y}{\int_{A_{i}} \int d x d y} \quad i=1, \ldots, 8 .
$$

Fig. 7 presents the distribution of permeability of $y=9,000 \mathrm{ft}$ for the initial guess, the 10th iteration for the steepest-descent version of the optimal control method, and the sixth iteration for the

TABLE 1 - COMPARISON OF HISTORY-MATCHING RESULTS USING TWO CONSTANT-ZONE METHODS AND TWO METHODS BASED ON THE OPTIMAL CONTROL ALGORITHM

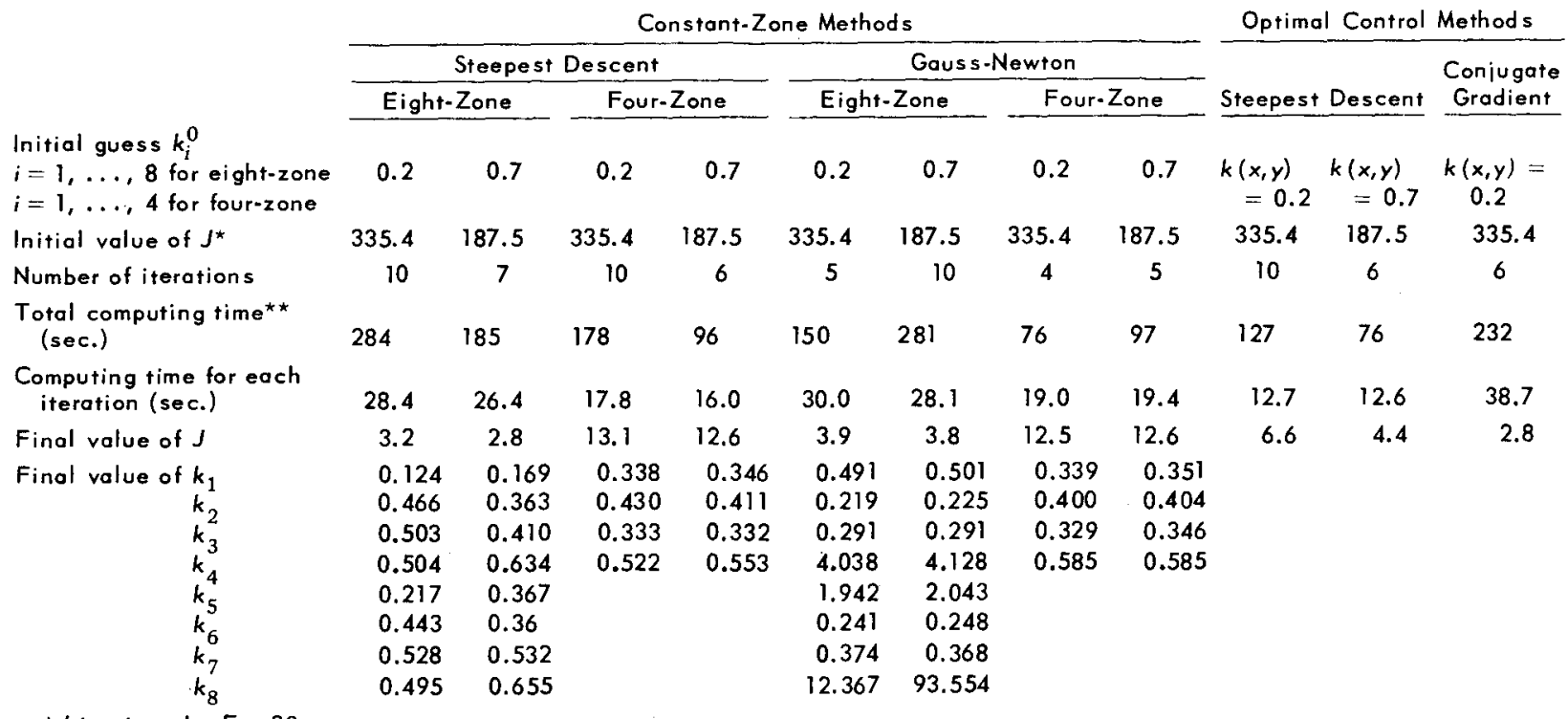


conjugate gradient version of the optimal control approach. Although in a one-dimensional reservoir case (the results are not shown here) the permeability distributor estimated by using the conjugate gradient method is closer to the assumed distribution than that obtained by the steepest-descent version of the optimal control method, there is no significant difference in the permeability distribution obtained by the steepest-descent method and by the conjugate gradient version in this example. From Table 1 we can see that the conjugate gradient method requires more computing time than the steepest-descent method to reach a comparable reduction in the value of $J$. This is because in the use of the conjugate gradient method one must calculate the objective function several times in each iteration in the use of a one-dimensional search technique to determine the optimal step length in each iteration. From this particular example we can conclude only that the conjugate gradient method appears to require somewhat more computing time than the steepestdescent version of the optimal control algorithm to reach a comparable reduction in $J$, but almost certainly offers better performance as the minimum is approached.

We can draw some interesting conclusions from the results in Table 1 and Figs. 3 through 7 . We note that in each case the least-squares criterion $J$ has been reduced significantly in a few iterations;



FIG. 3 - ACTUAL AND ESTIMATED PRESSURES AT WELL 2 FOR THE RESERVOIR OF FIGS. 1 AND 2. THE VARIATIONAL METHOD CURVE CORRESPONDS TO THE STEEPEST-DESCENT VERSION OF THE OPTIMAL CONTROL ALGORITHM.

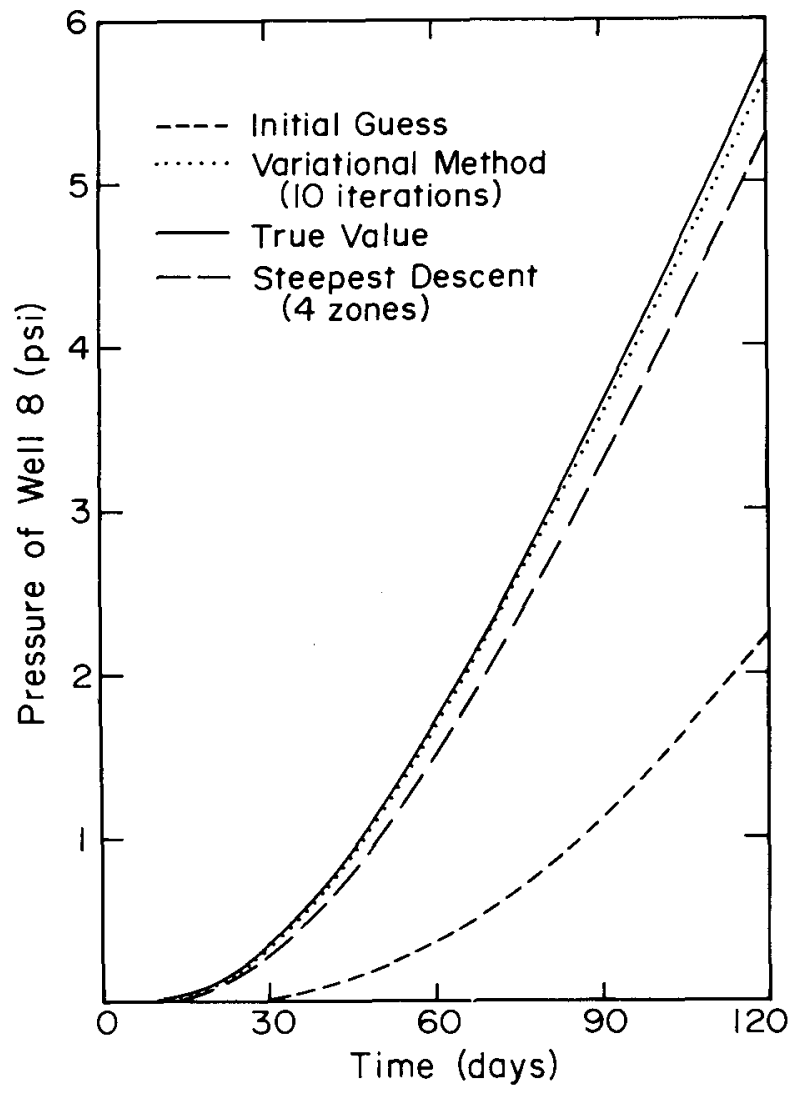

FIG. 4 - ACTUAL AND ESTIMATED PRESSURE AT WELL 8 FOR THE RESERVOIR OF FIGS。1 AND 2. THE VARIATIONAL METHOD CURVE CORRESPONDS TO THE STEEPEST-DESCENT VERSION OF THE OPTIMAL CONTROL ALGORITHM.

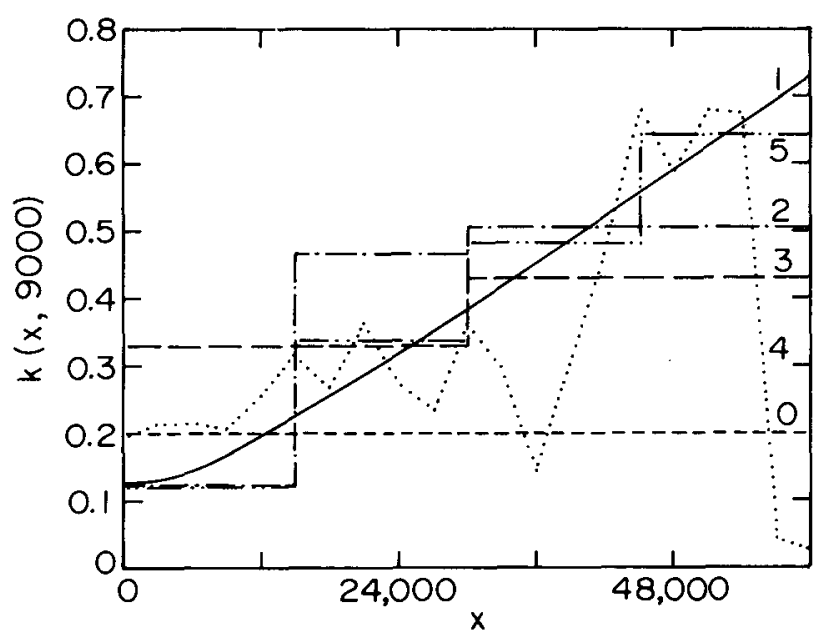

FIG. 5 - PERMEABILITY DISTRIBUTIONS AS A FUNCTION OF $x$ AT $y=9,000$ FT ESTIMATED FOR THE RESERVOIR OF FIGS. 1 AND 2.

\footnotetext{
$0=$ INITIAL GUESS

$1=$ TRUE VALUE

2 = STEEPEST DESCENT (EIGHT ZONES)

3 = STEEPEST DESCENT (FOUR ZONES)

$4=$ STEEPEST-DESCENT VERSION OF THE OPTIMAL CONTROL ALGORITHM

5 = AVERAGE VALUE (EIGHT ZONES)
} 
likewise, the pressure histories after a few iterations are very close to the observed pressures. Nevertheless, there is little correspondence between the final values of $k$ in the eight zones in the two constant-zone cases, as can be seen in Table 1 . Therefore, in spite of the fact that the four methods perform well in decreasing $J$, the parameter values determined by the separate methods bear no real relation to each other. The key problem is that the pressure histories at the observation wells are relatively insensitive to variations in $k(x, y) .12$ This becomes particularly apparent when the reservoir is divided arbitrarily into zones - zones that may bear no relation to the true $k(x, y)$ form in the reservoir; and the final $k$ values in these zones differ, depending on the experiment. More precisely, the reservoir system is relatively underdetermined

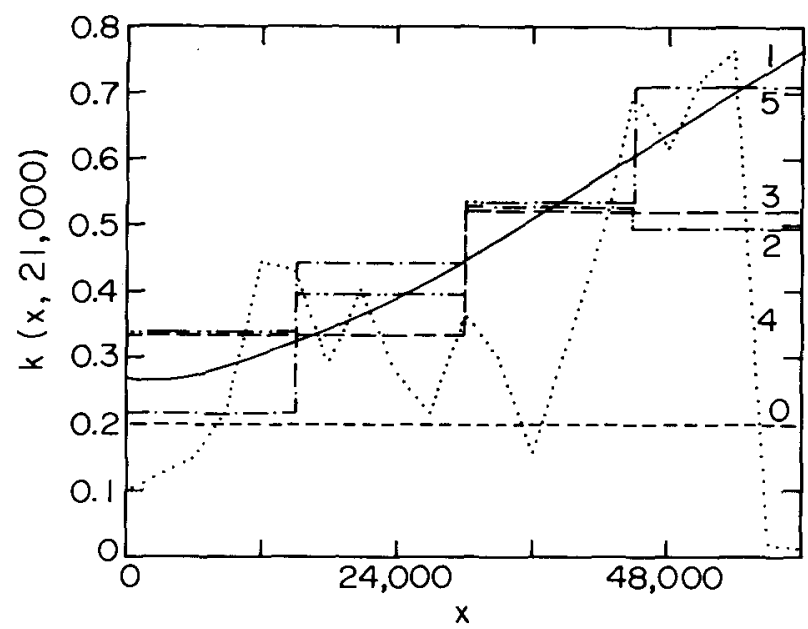

FIG. 6 - PERMEABILITY DISTRIBUTIONS AS A FUNCTION OF $x$ AT $y=21,000$ FT ESTIMATED FOR THE RESERVOIR OF FIGS. 1 AND 2. CURVES LABELED THE SAME AS IN FIG. 5.

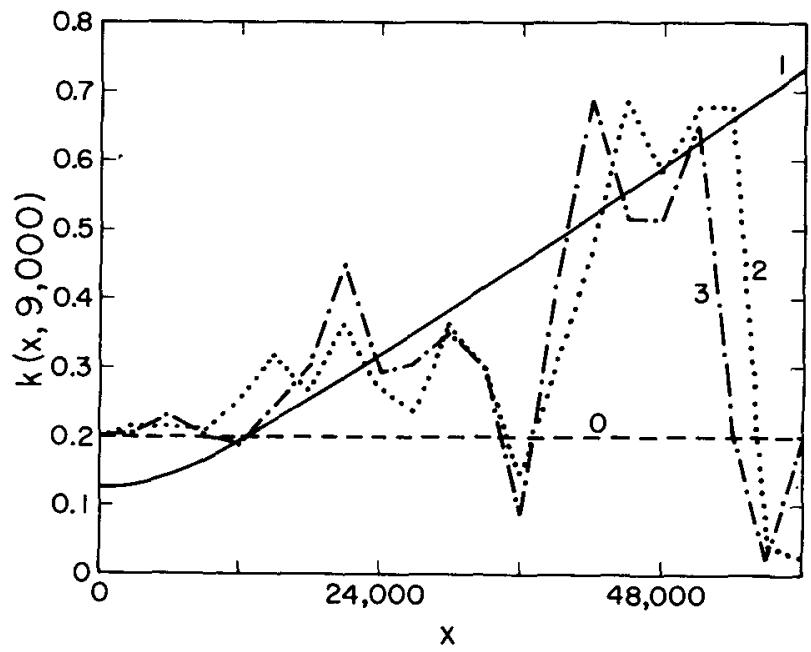

FIG. 7 - PERMEABILITY DISTRIBUTIONS AS A FUNCTION OF $x$ AT $y=9,000$ FT ESTIMATED FOR THE RESERVOIR OF FIGS. 1 AND 2.

$0=$ INITIAL GUESS

1 = TRUE VALUE

2 = STEEPEST-DESCENT VERSION OF THE OPTIMAL CONTROL ALGORITHM

3 - CONJUGATE GRADIENT VERSION OF THE DPTINAL CONTROL ALGORITHM with respect to $k(x, y)$.

\section{EXAMPLE 2}

The second example that we consider is the actual reservoir depicted in Fig. 8. Each grid block represents a distance of $1 \mathrm{~km}$, and each solid dot represents a producing well at which pressure data are a vailable over a period of roughly 10 years. The indicated boundary of the reservoir represents an oil/water interface, which did not move appreciably over the period for which data are available. Thus, the enclosed region in Fig. 8 can be considered as a single phase of oil, and the outer region can be taken as a single phase of water. The pressure behavior in each region is governed by a single-phase equation, where only the fluid viscosities and compressibilities are different in the two regions. The simulator equations were solved on the $23-x$ 26-km grid shown in Fig. 8.

The parameters to be estimated were the two transmissibilities in the horizontal directions, $k_{x} b$ and $k_{y} h$, and the storage coefficient, $\phi b$. These three parameters had been estimated previously by other history-matching methods. The parameters determined previously served as initial guesses for the steepest-descent version of the optimal control algorithm. Data were available at each of the 11 wells, a total of 118 pressure data points. Fig. 9 shows those grid cells in which changes of $k_{x} b$, $k_{y} b$, or $\phi b$ greater than 10 percent occurred after 60 iterations. For example, in Grid Block $(12,12)$ the initial guesses of $k_{x} b, k_{y} b$, and $\phi b$ were multiplied by $0.88,0.80$, and 0.89 , respectively. Fig. 10 shows a comparison of observed and calculated pressures at Well $A$ corresponding to the initial guesses of the parameters. Fig. 11 shows the observed and calculated pressures at Well $A$ after 60 iterations. Figs. 12 and 13 show the corresponding results for
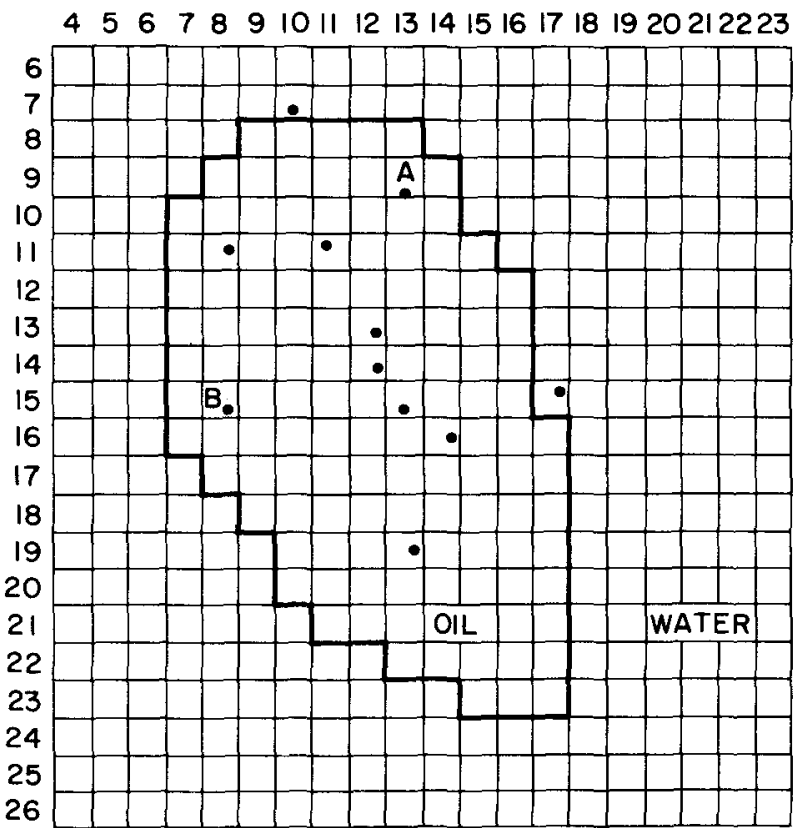

FIG. 8 - SAUDI ARABIAN RESERVOIR, EACH GRID BLOCK REPRESENTS A DISTANCE OF $1 \mathrm{KM}$. 
Well $B$. The initial root-mean-square values of the errors between observed and predicted pressures at Wells $A$ and $B$ were 84.9 and 188.9 , respectively. After 60 iterations, these values were reduced to 23.8 and 39.2 , respectively.

Some statistical properties of the history match are given in Table 2. The discrepancy between the observed and predicted pressures is represented by $\epsilon_{i}, i=1,2, \ldots, 118$. Check runs using Chevron Oil Field Research Co.'s multiphase reservoir simulator (CRS3D) were made after Iterations 0, 35, and 60 . In each case there was good pressure value agreement between the optimal control program and Chevron's program. One iteration of the steepestdescent optimal control method required $4.5 \mathrm{cpu}$ minutes. One run of Chevron's CRS3D program required $19 \mathrm{cpu}$ minutes - this would be the cost of one hand history-match run.

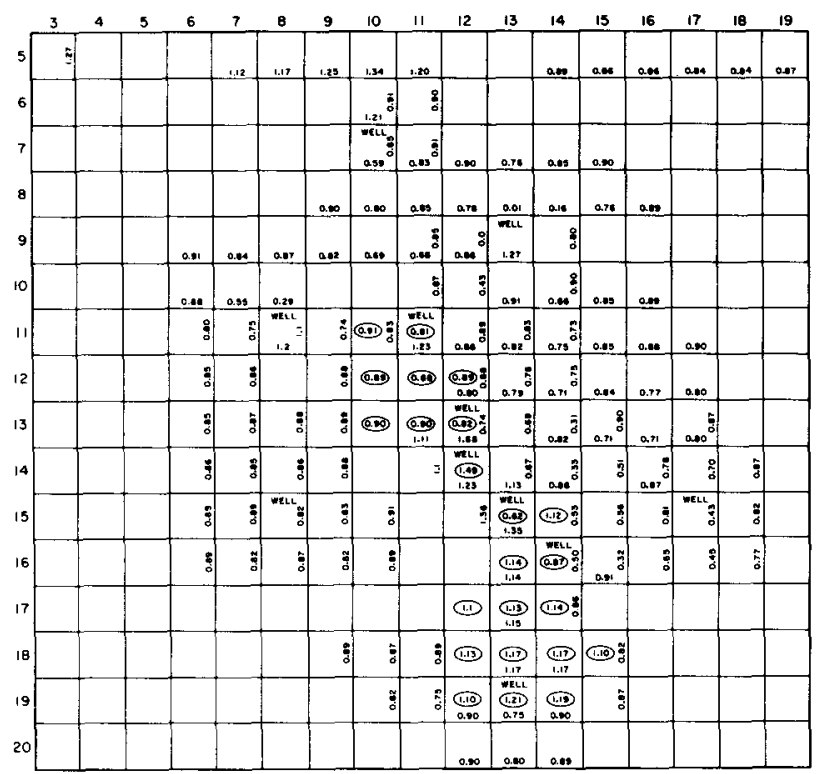

FIG. 9 - GRID CELLS IN WHICH CHANGES OF $k_{x} b$, $k_{V} h$, OR $\phi b$ GREATER THAN 10 PERCENT OCCURRED AFTER 60 ITERATIONS WITH THE STEEPESTDESCENT VERSION OF THE OPTIMAL CONTROL ALGORITHM.

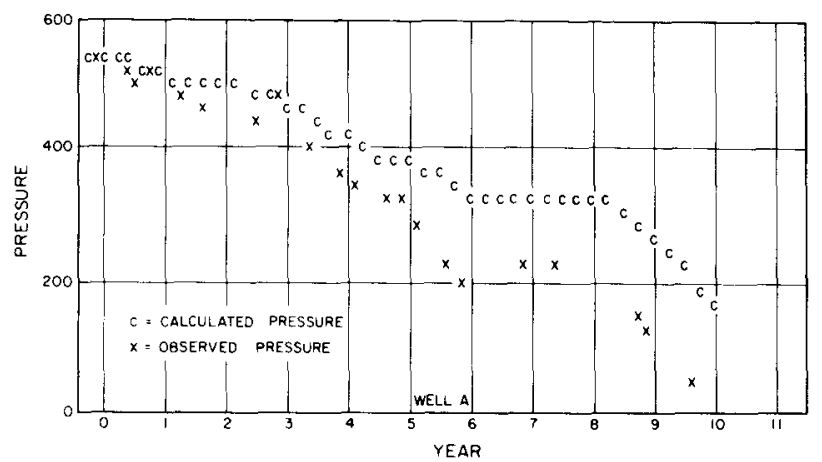

FIG. 10-OBSERVED AND CALCULATED PRESSURES AT WELL A CORRESPONDING TO THE INITIAL GUESSES OF THE PARAMETERS. THE PRESSURE SCALE SHOWN IS THAT RELATIVE TO AN ARBITRARY DATUM.

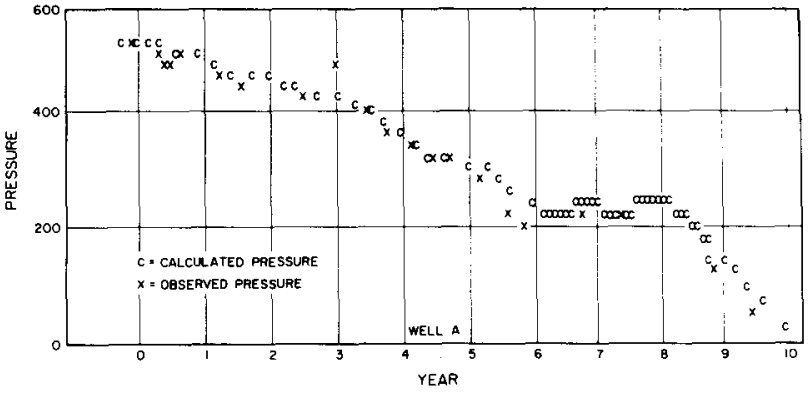

FIG. 11-OBSERVED AND CALCULATED PRESSURES AT WELL A AFTER 60 ITERATIONS. THE PRESSURE SCALE SHOWN IS THAT RELATIVE TO AN ARBITRARY DATUM.

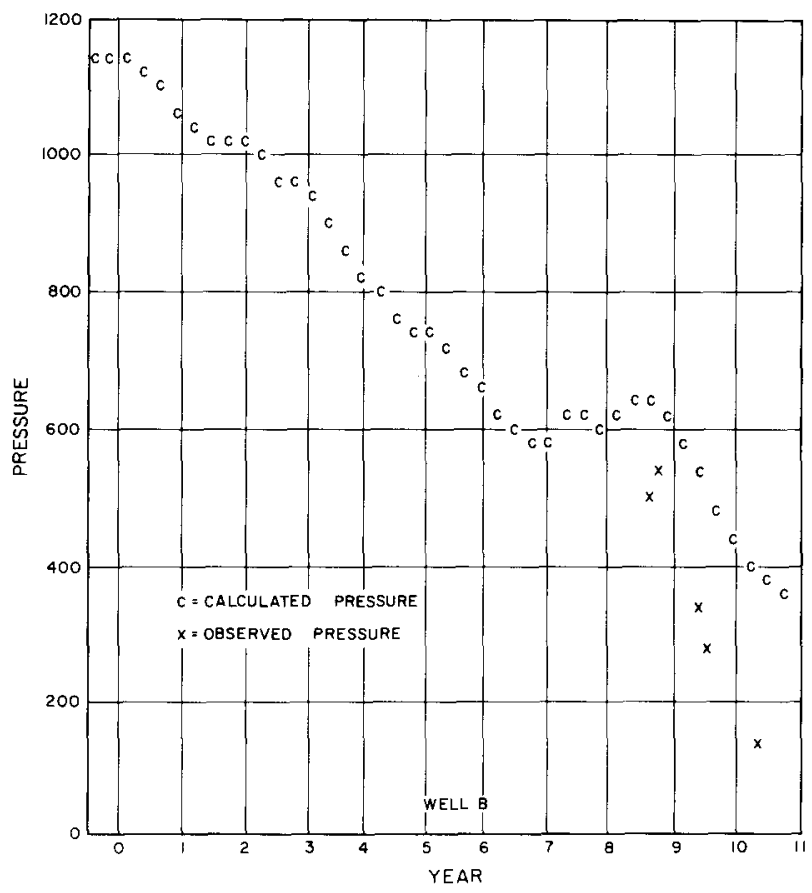

FIG. 12-OBSERVED AND CALCULATED PRESSURES AT WELL B CORRESPONDING TO THE INITIAL GUESSES OF THE PARAMETERS, THE PRESSURE SCALE SHOWN IS THAT RELATIVE TO AN ARBITRARY DATUM.

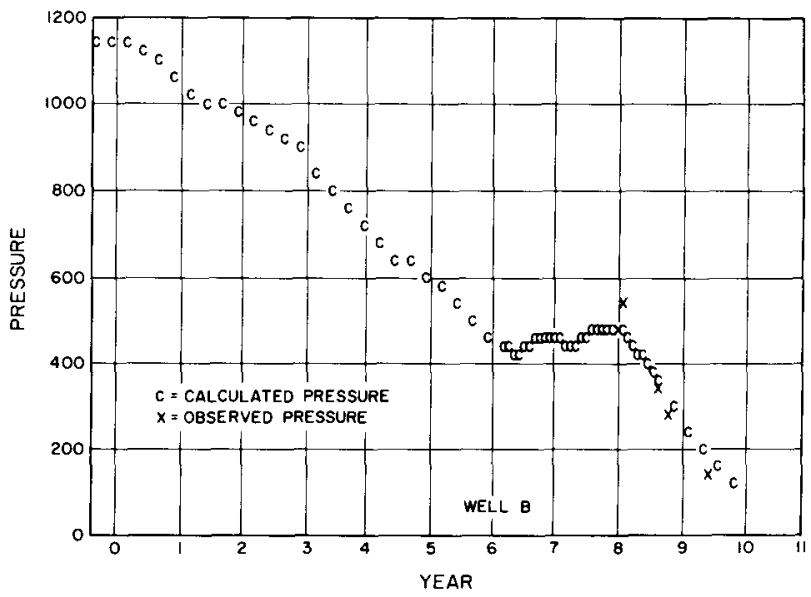

FIG. 13-OBSERVED AND CALCULATED PRESSURES AT WELL B AFTER 60 ITERATIONS. THE PRESSURE SCALE SHOWN IS THAT RELATIVE TO AN ARBITRARY DATUM. 


\section{CONCLUSIONS}

A new history-matching method designed to overcome the excessive computational requirements of standard methods is presented. The key features of the method are (1) that the reservoir property being estimated is treated as a continuous function of location rather than as one assuming discrete values in a number of zones and (2) that the history-matching problem is formulated as an optimal control problem. In a hypothetical exercise and for an actual reservoir the performance of the new method compared well with that of standard constant-zone gradient methods in reducing the least-squares performance index, but the new method required less computing time. Whereas the computing time for standard constant-zone methods usually increases directly either with the number of zones (or parameters) or with the number of pressure data points because of the required sensitivity coefficients, the computing time for the optimal control approach presented here does not change since constant zones are not employed. Thus, this new approach has promise of becoming the preferred standard method of performing all history-matching studies.

\section{NOMENCLATURE}

$A=$ defined by Eq. B-5

$A_{1}=\partial A / \partial p_{c}$

$A_{2}=\partial A / \partial p_{w}$

$a=N E S_{w}^{\prime}-N S_{w} E^{\prime}$

$a_{j i}=$ sensitivity coefficient defined by Eq. 3

$B_{e}=$ external boundary

$B_{w j}=$ boundary of $j$ th well

$B_{w}, B_{n}=$ formation volume factors for wetting and nonwetting phases

$b=M S_{w} E^{\prime}-N E S_{w}^{\prime}$

$c=$ compressibility

$D=$ subsea depth

$E=$ defined by Eq. B-6

$G=$ defined by Eq. B-7

$g=$ acceleration due to gravity

$H=$ Hessian matrix

$H_{i \ell}=$ element of $H$ defined by Eq. 39

$b=$ thickness of reservoir

$I=$ total number of measurements

$J=$ objective function defined by Eq. 1

$J^{j}=$ objéctive function at $j$ th iteration

TABLE 2 - PROPERTIES OF THE HISTORY MATCH

$$
\epsilon_{i}=p_{i}^{\text {obs }}-p_{i}
$$
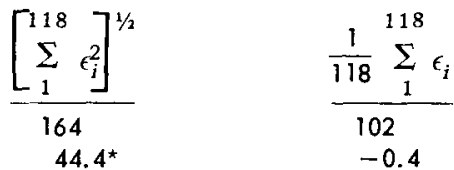

Initial guess

60 iterations $k(r), k(x, y)=$ permeabilities

$k_{i}=$ permeability at zone $i$

$k^{j}=$ permeability at $j$ th iteration

$k_{r w}, k_{r n}=$ relative permeabilities for wetting phase and nonwetting phase

$k_{x}=$ permeability in $x$-direction

$k_{y}=$ permeability in $y$-direction

$k^{0}=$ initial guess of permeability

$k^{*}=$ optimal estimate of permeability

$L=$ number of production wells

$L_{1}=$ length of a rectangular reservoir

$L_{2}=$ width of a rectangular reservoir

$\ell=$ coordinate along periphery of well

$M=$ number of observation locations

$M=$ (in Appendix B) $A_{1}-S_{w} A_{1}-A S_{w}^{\prime}+G S_{w}^{\prime}$

$N=$ number of zones

$N=$ (in Appendix B) $A_{2}-S_{w} A_{2}+S_{w} G^{\prime}$

$n=$ coordinate along outward normal

$n_{j}=$ number of observation data at $j$ th well

$p(r, t)=$ pressure

$p_{c}=$ capillary pressure

$p^{\mathrm{cal}}\left(r_{j}, t_{i}\right)=$ calculated pressure at $j$ th well at time $t_{i}$

$p^{\text {obs }}\left(r_{j}, t_{i}\right)=$ observed pressure at $j$ th well at time $t_{i}$

$p_{w}, p_{n}=$ pressures of wetting phase and nonwetting phase

$p^{0}(r, t)=$ initial guess of pressure

$p_{0}(\stackrel{r}{)}=$ initial pressure distribution

$q_{j}=$ flow rate of $j$ th well

$\hat{q}_{w}, \hat{q}_{n}=$ flow rate of wetting phase and nonwetting phase

$R_{i \ell}=$ element of Matrix $R$ defined by Eq. 38

$R_{s}=$ gas solubility

$\underline{r}=$ spatial coordinates

$\underline{r}_{j}=\left(x_{j}, y_{j}\right)=$ location of $j$ th well

$r_{w j}=$ radius of $j$ th well

$S=$ spatial domain

$S_{j}=$ conjugate gradient at $j$ th iteration

$S_{w}, S_{n}=$ saturations of wetting phase and nonwetting phase

$T=$ time period over which the observations are available

$t=$ time

$W(r)=$ weighting function

$x, y=$ spatial coordinates

$\alpha=$ hydraulic diffusivity

$\beta_{j}=$ scalar constant at $j$ th iteration

$\gamma=$ constant

$\delta()=$. Dirac delta function

$\epsilon=$ convergence criterion

$\epsilon_{j}=$ difference between the observed pressure and the calculated pressure of $j$ th observation

$\mu=$ viscosity

*After 35 iterations the root-mean-square error was 46.5 . 
$\mu_{w}, \mu_{n}=$ viscosities of wetting phase and nonwetting phase

$\rho_{w}, \rho_{n}=$ densities of wetting phase and nonwetting phase

$\phi=$ porosity

$\psi=$ adjoint variable

\section{ACKNOWLEDGMENT}

This work was supported in part by a grant from the Chevron Oil Field Research Co. Acknowledgment is given to Aramco for permission to publish the results of the Saudi Arabian reservoir study.

\section{REFERENCES}

1. Jacquard, P., and Jain, C.: "Permeability Distributions from Field Pressure Data," Soc. Pet. Eng. J. (Dec. 1965) 281-294; Trans., AIME, Vol. 234.

2. Jahns, H. O.: "A Rapid Method for Obtaining a Two-Dimensional Reservoir Description from Well Pressure Response Data," Soc. Pet. Eng. J. (Dec. 1966) 315-327; Trans., AIME, Vol. 237.

3. Brill, J. P., Burgoyne, A. T., and Dixon, T. N.: "Numerical Simulation of Drillstem Tests as an Interpretation Technique," J. Pet. Tech. (Nov. 1969) 1413-1420.

4. Coats, K. H., Dempsey, J. R., and Henderson, J. H.: "A New Technique for Determining Reservoir Description from Field Performance Data,' Soc. Pet. Eng. J. (March 1970) 66-74; Trans., AIME, Vol. 249.

5. Slater, G. E., and Durrer, E. J.: "Adjustment of Reservoir Simulation Models to Match Field Performance," Soc. Pet. Eng. J. (Sept. 1971) 295-305; Trans., AIME, Vol. 251.

6. Thomas, L. K., Hellums, L. J., and Reheis, G. M.: "A Nonlinear Automatic History Matching Technique for Reservoir Simulation Models," Soc. Pet. Eng. J. (Dec. 1972) 508-514; Trans., AIME, Vol. 253.

7. Veatch, R. W., Jr., and Thomas, G. W.: "A Direct Approach for History Matching," paper SPE 3515 presented at SPE-AIME 46th Annual Fall Meeting, New Orleans, La., Oct. 3-6, 1971.

8. Carter, R. D., Kemp, L. F., Jr., Pierce, A. C., and Williams, D. L.: "Performance Matching With Constraints," Soc. Pet. Eng. J. (April 1974) 187-196; Trans., AIME, Vol. 257.

9. Crockett, J. B., and Chernoff, H.: "Gradient Methods of Maximization," Pacific Jour. Matb. (1955) Vol. 5, 33.

10. Bard, Y.: "Comparison of Gradient Methods for the Solution of Nonlinear Parameter Estimation Problems," SIAM J. Numer. Anal. (1970) Vol. 7, 157.

11. Coats, K. H., and Terhune, M. H.: "Comparison of Alternating Direction Explicit and Implicit Procedures in Two-Dimensional Flow Calculations," Soc. Pet. Eng. J. (Dec. 1966) 350-362; Trans., AIME, Vo1. 237.

12. Dixon, T. N., Seinfeld, J. H., Startzman, R. A., and Chen, W. H.: "Reliability of Reservoir Parameters from History Matched Drillstem Tests," paper SPE 4282 presented at SPE-AIME Third Symposium on Numerical Simulation of Reservoir Performance, Houston, Jan. 10-13, 1973.

13. Snyder, L. J.: "Two-Phase Reservoir Flow Calculations," Soc. Pet. Eng. J. (June 1969) 170-182.
APPENDIX A

\section{NECESSARY CONDITIONS FOR OPTIMALITY}

In this appendix we derive the necessary conditions for optimality for the estimation of permeability in a single-phase reservoir model described by Eqs. 4 through 8. Although we shall consider here the estimation of $k(\tau)$, the method is general enough that other unknown properties - say porosity $\phi$ - could be treated similarly. The history-matching problem is to determine $k(r)$ to minimize Eq. 9. Since we assume that the pressure on the periphery of each well is constant, we can rewrite Eq. 9 as

$$
\begin{aligned}
J= & \sum_{j=1}^{M} \frac{1}{2 \pi r_{w_{j}}} \int_{0}^{T} \int_{B_{w_{j}}}\left[p^{\text {obs }}(r, t)\right. \\
& -\underset{\sim}{p(r, t)]^{2} d \ell d t . \ldots .}
\end{aligned}
$$

Concisely stated, we wish to minimize $J$, given by Eq. A-1, subject to Eqs. 4 through 8 . Using a variational approach we shall now derive the necessary conditions for optimality for this problem. Basically, we desire to relate a perturbation in $k(r), \delta k(r)$, to a perturbation in $J, \delta J$.

The change in $p(r, t), \delta p$, resulting from a change in $k(r)$, and hence $\alpha(r)$, is given by the solution of

$$
\left.\left.\frac{\partial \delta p}{\partial t}=\nabla \cdot(\delta \alpha \underset{\sim}{\alpha}) \nabla p\right)+\nabla \cdot(\underset{\sim}{\alpha} \underset{\sim}{r}) \nabla \delta p\right) .
$$

We introduce a so-called adjoint variable $\psi(r, t)$ and multiply Eq. A-2 by $\psi$ to give

$$
\begin{aligned}
& \frac{\partial}{\partial t}(\psi \delta p)=\frac{\partial \psi}{\partial t} \delta p+\psi \nabla \cdot(\delta \alpha \underset{\sim}{(r)} \nabla p) \\
& +\psi \nabla \cdot(\alpha \underset{\sim}{(r)} \nabla \delta p)
\end{aligned}
$$

Integrating both sides of Eq. A-3 over the spatial domain $S$ and over $t$ and using $\delta p(r, 0)=0$, we obtain

$$
\begin{aligned}
& \left.\iint_{S} \psi \delta p\right|_{T} d S=\int_{0}^{T} \int_{S} \int_{S} \frac{\partial \psi}{\partial t} \delta p d S d t \\
& +\int_{0}^{T} \int_{S} \psi \nabla \cdot\left(\underset{\sim}{\delta(r)} \int_{\sim} p p\right) d S d t \\
& +\int_{0}^{T} \int_{S} \psi \nabla \cdot \underset{\sim}{(\alpha(r) \nabla \delta p) d S d t} .
\end{aligned}
$$


By using the identity

$$
\nabla \cdot(U A)=(\nabla U) \cdot A+U \nabla \cdot A
$$

Eq. A-4 becomes

$$
\begin{aligned}
& \left.\iint_{S} \psi \delta p\right|_{T} d S=\int_{0}^{T} \iint_{S}\left\{\frac{\partial \psi}{\partial t} \delta p\right. \\
& +\nabla \cdot(\psi \delta \alpha(r) \nabla p)-(\nabla \psi) \cdot(\delta \alpha(r) \nabla p) \\
& +\nabla \cdot(\psi \alpha(r) \nabla \delta p)-\nabla \cdot\left(\delta p_{\alpha}(r) \nabla \psi\right) \\
& +\delta p \nabla \cdot(\alpha \underset{\sim}{(r) \nabla \psi)}\} \mathrm{dSdt}
\end{aligned}
$$

Employing the divergence theorem, we obtain

$$
\begin{aligned}
& \left.\iint_{S} \psi \delta p\right|_{T} d S=\int_{0}^{T} \int_{S} \int_{S} \frac{\partial \psi}{\partial t} \delta p d S d t \\
& +\int_{0}^{T} \int_{B_{e}} \psi \delta \alpha(r) \frac{\partial p}{\partial n} d \ell d t
\end{aligned}
$$$$
-\sum_{j=1}^{M} \int_{B_{w_{j}}}^{T} \psi \delta \alpha(\underset{\sim}{r}) \frac{\partial p}{\partial n} d \ell d t
$$$$
-\int_{0}^{T} \int_{S} \int_{S}(\nabla \psi \cdot \nabla p) \delta \alpha(\underset{\sim}{r}) d S d t
$$$$
+\int_{0}^{T} \int_{B_{e}} \psi \alpha \underset{\sim}{r} \frac{\partial \delta p}{\partial n} d \ell d t
$$$$
-\sum_{j=1}^{M} \int_{0}^{T} \int_{B_{w_{j}}} \psi \alpha(r) \frac{\partial \delta p}{\partial n} d \ell d t
$$$$
+\int_{0}^{T} \int_{S} \delta p \nabla \cdot(\alpha(r) \nabla \psi) d S d t
$$$$
-\int_{0}^{T} \int_{B_{e}} \delta p_{\alpha}(r) \frac{\partial \psi}{\partial n} d \ell d t
$$$$
+\sum_{j=1}^{M} \int_{0}^{T} \int_{B_{w_{j}}} \delta p \alpha(\underset{\sim}{r}) \frac{\partial \psi}{\partial n} d \ell d t .
$$

From Eq. 7 we know that

$$
\frac{\partial \delta \mathrm{p}}{\partial \mathrm{n}}=0 \quad \stackrel{\sim}{\sim} \in \mathrm{B}_{\mathrm{e}} .
$$

Using Eqs. 7 and A-8, Eq. A-7 can be written as

$$
\begin{aligned}
& \left.\iint_{S} \psi \delta p\right|_{T} d s=\int_{0}^{T} \iint_{S}\left\{\left[\frac{\partial \psi}{\partial t}+\nabla \cdot(\underset{\sim}{(r)} \nabla \psi)\right]\right. \\
& \cdot \delta p-(\nabla \psi \cdot \nabla p) \delta \alpha(r)\} d S d t \\
& -\int_{0}^{T} \int_{B_{e}} \alpha(r) \frac{\partial \psi}{\partial n} \delta p d \ell d t-\sum_{j=1}^{M} \int_{0}^{T} \int_{B_{W_{j}}} \\
& \text { - } \psi\left[\underset{\sim}{\alpha} \underset{\sim}{r} \frac{\partial \delta p}{\partial n}+\delta \alpha \underset{\sim}{(r)} \frac{\partial p}{\partial n}\right] d \ell d t \\
& +\sum_{j=1}^{M} \int_{0}^{T} \int_{B_{w_{j}}} \alpha(r) \frac{\partial \psi}{\partial n} \text { spdedt. }
\end{aligned}
$$

The change in $J, \delta J$, resulting from a change in $p(r, t)$ is given by

$$
\begin{aligned}
\delta J=- & \sum_{j=1}^{M} \frac{1}{\pi r_{w_{j}}} \int_{0}^{T} \int_{B_{w_{j}}}\left[p^{\text {obs }} \underset{\sim}{r}, t\right) \\
& -p(r, t)] \delta p d \ell d t \ldots(A
\end{aligned}
$$

Combining Eqs. A-9 and A-10 gives

$$
\begin{aligned}
& \left.\iint_{S} \psi \delta p\right|_{T} d S+\delta J=\int_{0}^{T} \iint_{S}\left\{\frac{\partial \psi}{\partial t}\right. \\
& +\nabla \cdot(\alpha \underset{\sim}{(r)} \nabla \psi) \delta p-(\nabla \psi \cdot \nabla p) \delta \alpha(r)\} d S d t \\
& \int_{0}^{T} \int_{B_{e}} \alpha \underset{\sim}{(r)} \frac{\partial \psi}{\partial n} \delta p d \ell d t \\
& -\sum_{j=1}^{M} \int_{0}^{T} \int_{B_{W}} \psi\left[\underset{\sim}{\alpha \underset{\sim}{r})} \frac{\partial \delta p}{\partial n}+\delta \alpha \underset{\sim}{(r)} \frac{\partial p}{\partial n}\right] d \ell d t \\
& +\sum_{j=1}^{M} \int_{0}^{T} \int_{B_{w_{j}}}^{j}\left\{\underset{\sim}{j} \alpha(r) \frac{\partial \psi}{\partial n}-\frac{1}{\pi r_{w_{j}}}\left[p^{o b s}(\underset{\sim}{r}, t)\right.\right.
\end{aligned}
$$




$$
-\underset{\sim}{p(r, t)]}\} \delta p d \ell d t
$$

The object of adding $J$ to Eq. A-9 is to obtain a relation between $\delta J$ and $\delta a(r)$. To determine the relation between $\delta J$ and $\delta \alpha(r)$, let us have $\psi(r, t)$ be governed by

$$
\left.\frac{\partial \psi}{\partial t}=-\nabla \cdot \underset{\sim}{(\alpha)} \underset{\sim}{(r)}\right)
$$

with the final condition

$$
\psi(r, T)=0
$$

and choose the boundary conditions for Eq. A-12 so that the second, third, and fourth terms on the right-hand side of Eq. A-11 vanish. This can be done as follows. Because of the arbitrariness of $\delta p$ on the boundaries and the uniformity of $\delta p$ on the inner boundary, $B_{w j}, j=1, \ldots, M$

$$
\int_{0}^{T} \int_{B_{e}} \alpha(r) \frac{\partial \psi}{\partial n} \delta p d \ell d t=0,
$$

and

$$
\begin{aligned}
& \sum_{j=1}^{M} \int_{0}^{T} \int_{B_{w_{j}}}\left\{\alpha(r) \frac{\partial \psi}{\partial n}-\frac{1}{\pi r_{w_{j}}}\left[p^{o b s} \underset{\sim}{(r, t)}\right.\right. \\
& \quad-\underset{\sim}{p(r, t)]\} \delta p d \ell d t=0,}
\end{aligned}
$$

imply

$$
\frac{\partial \psi}{\partial n}=0
$$$$
r \varepsilon \mathrm{B}_{\mathrm{e}}
$$

and

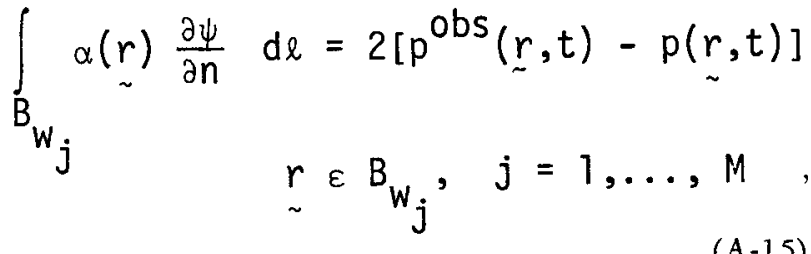

respectively. To find the third boundary condition, we need only note that from Eq. 6

$$
\begin{array}{r}
\int_{B_{W_{j}}}\left[\alpha(r) \frac{\partial \delta p}{\partial n}+\delta \alpha(r) \frac{\partial p}{\partial n}\right] d l=0 \\
\underset{\sim}{r} \varepsilon B_{W_{j}}, j=1, \ldots, M, .(A)
\end{array}
$$

and that in order for the relation

$$
\begin{gathered}
\left.\sum_{j=1}^{M} \int_{0}^{T} \int_{B_{W_{j}}} \psi\left[\underset{\sim}{\alpha} \underset{\sim}{r} \frac{\partial \delta p}{\partial n}+\delta \alpha \underset{\sim}{r}\right) \frac{\partial p}{\partial n}\right] d \ell d t \\
=0
\end{gathered}
$$

to hold, a sufficient condition is that

$$
\frac{\partial \psi}{\partial l}=0 \quad r \varepsilon B_{w_{j}}, j=1, \ldots, M
$$

This is the third boundary condition.

Substituting Eqs. A-12 through A-15 and Eq. A-17 into Eq. A-11 we obtain

$$
\delta J=-\int_{0}^{T} \iint_{S}[(\nabla \psi) \cdot(\nabla p)] \delta \alpha(r) d S d t .
$$

We can interchange the order of integration as follows:

$$
\delta J=-\iint_{S}\left\{\int_{0}^{T}[(\nabla \psi) \cdot(\nabla p)] d t\right\} \delta \alpha(\underset{\sim}{r}) d S .
$$

Finally, the condition that $\delta J=0$ is obtained if

$$
\int^{T}[(\nabla \psi) \cdot(\nabla p)] d t=0 \text {. }
$$

Summarizing, the necessary conditions for optimality are given by Eqs. 4 through $8, A-12$ through $A-15$, $A-17$, and $A-20$.

\section{APPENDIX B}

\section{HISTORY MATCHING FOR TWO-PHASE RESERVOIRS}

The flow of two partially miscible fluids in a porous medium is governed by the following partial differential equations ${ }^{13}$ :

$$
\begin{array}{r}
\nabla \cdot\left[\frac{k k_{W}}{\mu_{W} B_{W}}\left(\nabla p_{W}-\rho_{W} g \nabla D\right)\right]=\frac{\partial}{\partial t}\left(\frac{\phi S_{W}}{B_{W}}\right) \\
-\hat{q}_{w} \ldots \ldots
\end{array}
$$

and 


$$
\begin{aligned}
& \nabla \cdot\left[\frac{k k_{r n}}{\mu_{n} B_{n}}\left(\nabla p_{n}-\rho_{n} g \nabla D\right)+\frac{R_{s} k k_{r w}}{\mu_{w} B_{w}}\left(\nabla p_{w}\right.\right. \\
& \left.\left.-\rho_{w} g \nabla D\right)\right]=\frac{\partial}{\partial t}\left[\phi\left(\frac{S_{n}}{B_{w}}+\frac{R_{s} S_{w}}{B_{w}}\right)\right]-\hat{q}_{n} .
\end{aligned}
$$

The phase pressures are related through the capillary pressure, which is a function of saturation

$$
p_{n}-p_{w}=p_{c}=f\left(S_{w}\right)
$$

The saturations are subject to the condition

$$
s_{n}+s_{w}=1
$$

Eqs. B-1 through B-4, together with appropriate initial and boundary conditions, form a common description of the behavior of two partially miscible fluids in a reservoir.

The history-matching problem of interest is to estimate $k$ and $\phi$. In this appendix we show how the system of Eqs. B-1 through B-4 can be rearranged so that the optimal control approach can be used directly to produce algorithms for the estimation of $k$ and $\phi$. For the purpose of deriving the desired form of Eqs. B-1 through B-4, we shall consider the dependent variables as $p_{w}$ and $p_{c}$. Let us define $S_{w}=f_{1}\left(p_{c}\right), S_{n}=1-S_{w}, p_{c}=p_{n}-p_{w}, k_{r w}=f_{2}\left(S_{w}\right)$, $k_{r n}=f_{3}\left(S_{n}\right), B_{w}=f_{4}\left(p_{w}\right), \mu_{w}=f_{5}\left(p_{w}\right), \rho_{w}=f_{6}\left(p_{w}\right)$, $R_{s}=f_{7}\left(p_{w}\right), B_{n}=f_{8}\left(p_{n}\right), \mu_{n}=f_{9}\left(p_{n}\right)$, and $\rho_{n}=f_{10}\left(p_{n}\right)$. Thus, we can express $k_{r w}$ and $k_{r n}$ as functions of $p_{c}$ and $B_{n}, \mu_{n}$, and $\rho_{n}$ as functions of $p_{C}$ and $p_{w}$. Let us write

$$
\begin{aligned}
& \frac{\phi}{B_{n}}=A\left(p_{C}, p_{W}\right), \\
& \frac{\phi}{B_{W}}=E\left(p_{W}\right), . \\
& \frac{R_{S} \phi}{B_{W}}=G\left(p_{W}\right) .
\end{aligned}
$$

Furthermore, we assume that capillary pressure data under imbibition conditions will uniquely describe the relationship between $p_{c}$ and $S_{w}$. Since $p_{c}$ and $S_{w}$ are uniquely related, $\partial S_{w} / \partial t$ can be expressed as

$$
\frac{\partial S_{W}}{\partial t}=\frac{d S_{W}}{d p_{C}} \frac{\partial p_{C}}{\partial t}=S_{W}^{\prime} \frac{\partial p_{C}}{\partial t} .
$$

$$
\begin{aligned}
& \nabla \cdot\left[\frac{k_{k} r_{w}}{\mu_{w} B_{w}}\left(\nabla p_{w}-\rho g \nabla D\right)\right]=E S_{w}^{\prime} \frac{\partial p_{C}}{\partial t} \\
& +S_{W} E^{\prime} \frac{\partial p_{W}}{\partial t}-\hat{q}_{W} \\
& \nabla \cdot\left[\frac{k k_{r n}}{\mu_{u} B_{n}}\left(\nabla\left\{p_{c}+p_{w}\right\}-\rho_{n} g \nabla D\right)\right. \\
& \left.+\frac{R_{s} k k_{w w}}{\mu_{w}{ }^{B} w}\left(\nabla p_{w}-\rho_{w} g \nabla D\right)\right] \\
& =\left(A_{1}-S_{W} A_{1}-A S_{W}^{\prime}+G S_{W}^{\prime}\right) \frac{\partial p_{C}}{\partial t} \\
& +\left(A_{2}-S_{w} A_{2}+S_{W} G^{\prime}\right) \frac{\partial p_{w}}{\partial t}-\hat{q}_{n},
\end{aligned}
$$

where $A_{1}$ and $A_{2}$ denote $\partial A / \partial p_{c}$ and $\partial A / \partial p_{w}$ respectively.

We rewrite Eqs. B-9 and B-10 as

$$
\begin{aligned}
E S_{W}^{\prime} & \frac{\partial p_{C}}{\partial t}+S_{W} E^{\prime} \frac{\partial p_{W}}{\partial t}= \\
\nabla & {\left[\frac{k k_{W}}{\mu_{W} B_{W}}\left(\nabla p_{W}-\rho_{W} g \nabla D\right)\right]+\hat{q}_{W}, . }
\end{aligned}
$$

$$
\begin{aligned}
& M \frac{\partial p_{c}}{\partial t}+N \frac{\partial p_{w}}{\partial t}=\nabla \cdot\left[\frac { k k _ { r n } } { \mu _ { n } B _ { n } } \left(\nabla\left\{p_{c}+p_{w}\right\}\right.\right. \\
& \left.\left.-\rho_{n} g \nabla D\right)+\frac{R_{s} k k_{r w}}{\mu_{w} B_{w}}\left(\nabla p_{w}-\rho_{w} g \nabla D\right)\right]+\hat{q}_{n}
\end{aligned}
$$

where $M=A_{1}-S_{w} A_{1}-A S_{w}^{\prime}+G S_{w}^{\prime}$ and $N=A_{2}-$ $S_{w} A_{2}+S_{w} G^{\prime}$

Multiplying Eq. B-11 by $N$, Eq. B-12 by $-S_{w} E^{\prime}$, and adding the resulting equations gives

$$
\begin{aligned}
\frac{\partial p_{c}}{\partial t} & =\frac{N}{a} \nabla \cdot\left[\frac{k k_{w}}{\mu_{w} B_{w}}\left(\nabla p_{w}-\rho_{w} g \nabla D\right)\right] \\
& -\frac{S_{w^{\prime}} E^{\prime}}{a} \nabla \cdot\left[\frac{k k_{r n}}{\mu_{n} B_{r}}\left(\nabla\left\{p_{C}+p_{w}\right\}-\rho_{n} g \nabla D\right)\right.
\end{aligned}
$$

Using Eqs. B-5 through B-7, Eqs. B-1 and B-2 become 


$$
\begin{gathered}
\left.+\frac{R_{s}{ }^{k}{ } w}{\mu_{w} B_{w}}\left(\nabla p_{w}-\rho_{w^{\prime}} g \nabla D\right)\right] \\
+\frac{N}{a} \hat{q}_{w}-\frac{S_{w^{\prime}} E^{\prime}}{a} \hat{q}_{n},
\end{gathered}
$$

where $a=N E S_{w}^{\prime}-M S_{w} E^{\prime}$. Multiplying Eq. B-11 by $M$, Eq. B-12 by $-E S_{w}^{\prime}$, and adding the resulting equations gives

$$
\frac{\partial p_{w}}{\partial t}=\frac{M}{b} \nabla \cdot\left[\frac{k k_{w}}{\mu_{w} B_{w}}\left(\nabla p_{w}-\rho_{w} g \nabla D\right)\right]
$$

$$
\begin{aligned}
& -\frac{E S_{W}^{\prime}}{b} \nabla \cdot\left[\frac{k k_{r n}}{{ }_{n} B_{n}}\left(\nabla\left\{p_{c}+p_{w}\right\}-\rho_{n} g \nabla D\right)\right. \\
& \left.+\frac{R_{s} k k_{r W}}{\mu_{W} B_{W}}\left(\nabla p_{W}-\rho_{w} g \nabla D\right)\right]
\end{aligned}
$$

$$
+\frac{M}{b} \hat{q}_{w}-\frac{E S_{w}^{\prime}}{b} \hat{q}_{n}, .
$$

where $b$ denotes $M S_{w} E^{\prime}-N E S_{w}^{\prime}$.

Once the system has been placed in the form of Eqs. B-13 and B-14 the procedure that was used in the development of optimal control algorithms for a single-phase reservoir in Appendix A can be applied to derive optimal control algorithms for estimating parameters in the multiphase reservoir model. 